\title{
An Ectopic Pelvic Kidney
}

Zhi Yang ${ }^{1,2}$, Qin Xiang ${ }^{1,2}$, Wei Liu ${ }^{1,2 *}$

${ }^{1}$ Institute of Digestive Disease, China Three Gorges University, Yichang, China.

${ }^{2}$ Department of Gastroenterology, Yichang Central People's Hospital, Yichang, China.

*Corresponding author: Wei Liu, Ph.D., Institute of Digestive Disease, China Three Gorges University, 8 Daxue Road, Yichang 443000, China.

Received date: March 29, 2021: Accepted date: April 08, 2021: Published date: April 12, 2021

Citation: Z Yang, Q Xiang, W Liu. (2021) An Ectopic Pelvic Kidney. Journal of Clinical Surgery and Research. 2(2); DOI:10.31579/2768$2757 / 010$

Copyright: (2021 Wei Liu, This is an open-access article distributed under the terms of the Creative Commons Attribution License, which permits unrestricted use, distribution, and reproduction in any medium, provided the original author and source are credited.

A 46-year-old man presented to the internal medicine department with a 1 -year history of abdominal pain. His medical history was nonobstructive hypertrophic cardiomyopathy. Physical examination was unremarkable. As part of the evaluation, computed tomography of abdomen was performed. Focused assessment with computed tomography confirmed no evidence of ascites, but the left kidney could not be identified. A whole-body computed tomography discovered a pelvic left kidney (Figure 1A). Renal function of the patient was normal with a creatinine level of $0.89 \mathrm{mg}$ per deciliter ( $77 \mu \mathrm{mol}$ per liter) (normal range, 0.5 to $1.1 \mathrm{mg}$ per deciliter [ 48 to $90 \mu \mathrm{mol}$ per liter]). It is a common incidental finding because the affected persons are commonly asymptomatic. Renal ectopia is commonly caused by failure of mature kidney to reach its natural location in renal fossa [1]. The uncommon congenital abnormality was related to the aberrant processes during embryogenesis [2]. Ectopic kidney may be found in pelvic, abdominal, iliac, and thoracic location, among which pelvic ectopia is estimated to occur in 1 of 3000 autopsies [3]. As in this case, the rare condition may become unknown until computed tomography imaging is performed for another reason. Finally, the patient's abdominal pain was attributed to the duodenal ulcer confirmed by esophagogastroduodenoscopy, and he was treated with a proton pump inhibitor. 


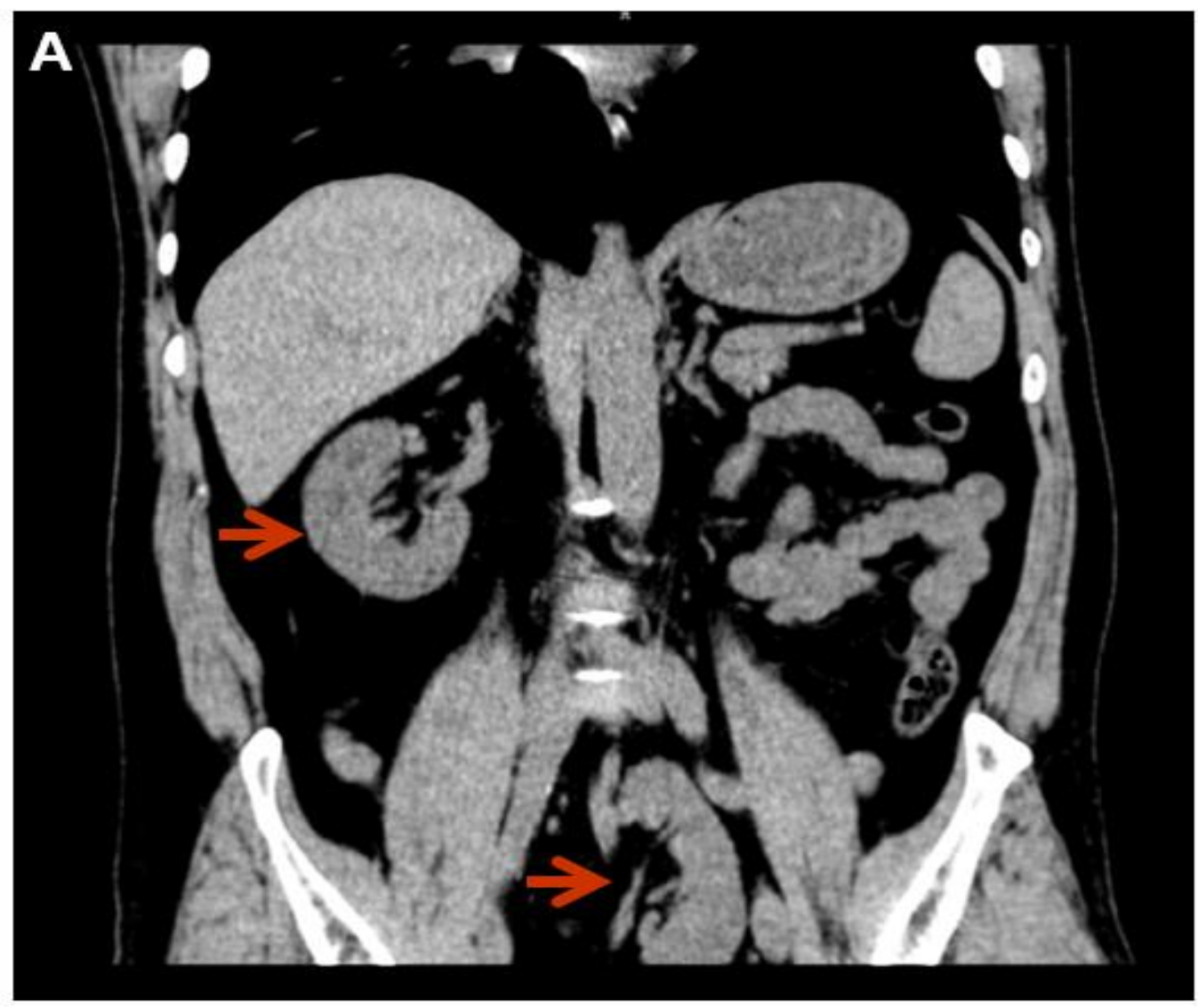

\section{Acknowledgements}

Funding: This work was supported by National Natural Science Foundation of China (31600134).

\section{Footnote}

Conflicts of Interest: The authors have no conflicts of interest to declare.

Ethical Statement: The authors are accountable for all aspects of the work in ensuring that questions related to the accuracy or integrity of any part of the work are appropriately investigated and resolved. Written informed consent was obtained from the patient for publication of this "Image of Month".

\section{Author's contributions}

Collection of data: Zhi Yang.

Manuscript preparation and writing: Qin Xiang.

Final approval of the manuscript: Wei Liu.

\section{References}

1. Almeida CC, Vaz AM. (2020) Ectopic Kidney. N Engl J Med. 383(5).

2. Bhoil R, Sood D, Singh YP, et al. (2015) An Ectopic Pelvic Kidney. Pol J Radiol. 80:425-427.

3. Eid S, Iwanaga J, Loukas M, et al. (2018) Pelvic Kidney: A Review of the Literature. Cureus. 10(6):e2775. 